\title{
Caractérisation de la Houle par des Surfaces de Réponse pour l'analyse en Fiabilité des Plates-Formes Pétrolières
}

\author{
Franck Schoefs \\ Allocataire Moniteur Normalien, L.M.G, Faculté des Sciences de Nantes \\ Jacques Labeyrie \\ Responsable Fonctionnel Recherche DITI, IFREMER, centre de Brest
}

\begin{abstract}
Résumé
Dans le contexte actuel de requalification des structures pétrolières "offshore" arrivant en date butée de durée d'exploitation, on se propose ici de mettre en place un modèle de champ cinématique pour la houle de Stokes en vue d'une utilisation dans un calcul de fiabilité. L'approche, de type "Surface de Réponse", permet de caractériser la typologie des champs cinématiques et de montrer que les fluctuations sont portées par des fonctions profil, tout en facilitant leur transfert pour le calcul d'efforts hydrodynamiques sur les barres d'une plate-forme de type "jacket". Les illustrations proviennent de simulations reposant sur des bases de données construites à partir de données in situ de mer du Nord.
\end{abstract}

\section{Introduction}

L'analyse en fiabilité des structures à la mer s'est généralisée ces dix dernières années, dès la phase de conception. Toutefois, c'est l'enjeu économique de la requalification des structures existantes qui suscite aujourd'hui une forte demande dans ce domaine. En effet, un grand nombre de plates-formes "offshore" implantées dans les années 70 étaient conçues pour une durée d'exploitation de 20 ans. Le secteur pétrolier doit donc se doter d'outils performants d'analyse en fiabilité, permettant notamment de définir des procédures d'inspection, afin de décider de la reconduction, la réhabilitation ou de la mise hors-service de la structure.

Toute procédure s'appuie sur des concepts de base et celui des surfaces de réponse est prépondérant pour l'analyse fiabiliste. Il s'agit d'appréhender un phénomène physique par une approche système c'est à dire comme la réponse à plusieurs stimuli. La représentation dans l'espace des variables stimuli, de la fonctionnelle d'approximation est appelée surface de réponse. Cette construction nécessite le respect de différents critères comme le sens physique sous-tendu par le modèle, le transfert des distributions de probabilité des variables de base, la dimensionnalité du problème, l'interdépendance des variables. L'étude présentée ici s'inscrit dans ce besoin de proposer des procédures robustes en vue de construire ces surfaces. 
La démarche retenue repose alors sur l'utilisation des lois déterministes et lintroduction d'aléa. Au cours du calcul fiabiliste, on effectue des transferts successifs, via ces lois (efforts répartis, nodaux, calcul de structure), de la surface de réponse d'otigine.

Nous proposons ici de construire la surface de réponse de base pour un calcul de fiabilité en quasi-statique, sous des conditions extrêmes d'environnement marin; il s'agit de caractériser la houle par sa cinématique en se basant sur le modèle déterministe de la houle de Stokes au n-ième ordre.

Une fois le formalisme mis en place, la réponse relative au champ de vitesse sera caractérisée par sa typologie sur un site de mer du Nord, le champ de Frigg, réputé pour ses conditions sévères.

Auparavant, il parait nécessaire de préciser les fondements et les objectifs de la méthodologie des surfaces de réponse.

\section{Construction d'une surface de réponse}

\subsection{Objectifs de l'analyse fiabiliste}

La finaité de lanalyse fiabiliste est d'estimer la valeur d'une mesure de la sécurité du dimensionnement de la structure par rapport à un point de dysfonctionnement situé à la limite des domaines acceptables et non acceptables (fonctionnalité non accomplie, ruine plastique, ...). Cette frontière, appelée surface de ruine et plus généralement surface d'état limite, rend compte de linteraction entre grandeurs résistantes et sollicitations. Une mesure de la sécurité peut alors être effectuée sous forme d'indice de fiabilité ou de probabilité de ruine [1].

Pour cela, sollicitations et résistances doivent être modélisées afin de prendre en compte leurs fluctuations en termes de variables aléatoires intrinsèques et de paramètres d'incertitude sur les modèles.

\subsection{Concepts de base et définitions des sarfiaces de réponse}

Lorsque l'on adopte la méthodologie des surfaces de réponse dans l'étude d'un phénomène physique; on cherche à déterminer des approximations (fonctions de réponse) permettant d'approcher le processus physique d'origine. En effet le transfert explicite des variables du problème à la réponse $Y$ (propriété étudiée) est en général inconnu. On suit alors une approche de type système en considérant la propriété étudiée comme la réponse de ce système de composants à des variables d'entrées "stimuli" représentatives du phénomène (variables de base).

La représentation de cette réponse dans l'espace des variables "stimuli" appelées variables de base est une (hyper)-surface de réponse. Pour atteindre cet objectif, il faut donc sélectionner :

- un ensemble fini de variable aléatoire stimuli $X_{i}$,

- des informations statistiques $\theta_{i}$ sur ces variables dites de base (fonction de distributions, moments normalisés...),

- $f$, une fonction d'approximation explicite des $X_{i}$ connaissant $\theta_{i}$.

- une métrique dans l'espace de $Y$ et de fafin de mesurer la qualité de l'ajustement. 
Les concepts et les fonctions usuelles utilisés dans des domaines aussi variés que la biologie, l'électronique et la sûreté nucléaire ont été plus largement développés par Mead et Pike [2]. Les problèmes relevant des systèmes mécaniques ont nécessité de développer des critères de construction spécifiques. Ainsi, le transfert des distributions, le respect du sens physique des phénomènes, la qualité de l'ajustement et l'adaptation au calcul informatique doivent être analysés et discutés avec soin pour sélectionner une surface de réponse fiable et robuste.

\subsection{Critères associés à la construction de surfaces de réponse}

La sélection de l'ensemble des variables de base et leur classement par ordre d'importance de leur contribution sont les premiers points délicats de la méthodologie. Cet ensemble représentatif du phénomène doit rester de dimension réduite (notion d'exhaustivité) et l'influence des hypothèses de corrélation/indépendance doit être analysée.

Le respect du sens physique et le transfert des distributions participent du même souci de représentativité physique de la fonction de réponse sélectionnée. Le phénomène physique doit être bien transcrit dans ses propriétés et la fonction de réponse, en terme de fonction de transfert, vise à rendre compte en sortie des particularités des distributions des variables [ 3 ].

Un autre groupe de critères reflète l'exigence de qualité : mesure de la qualité de l'ajustement et adaptation du modèle au calcul informatique (impératif d'ingénierie de limitation du temps de calcul). En particulier, le niveau de complexité de la modélisation doit être introduit avec une large réflexion : il influe de manière très sensible sur la durée du calcul et n'aboutit pas pour autant à des résultats nécessairement plus réalistes.

Lors de la conduite de simulations enfin, la même attention doit être accordée à la construction d'échantillons afin d'allier représentativité physique et adaptation au calcul informatique. La taille, l'étendu du support et la contribution dans la variabilité du modèle sont autant de questions à préciser.

Il apparait donc clairement que de nombreux points doivent être abordés et que le choix final ne peut résulter que d'une optimisation sous contraintes et en vue de fournir la fonction d'approximation $f$, deux approches basées sur cette méthodologie se distinguent :

- une démarche purement analytique de recherche de fonctions d'approximation mathématiques, en général sous forme polynomiale (travaux de Rackwitz et coll. [4] pour le calcul d'efforts hydrodynamiques sur une barre).

- une utilisation de modèles déterministes éprouvés et la prise en compte de fluctuations par l'introduction de variables aléatoires intrinsèques ou paramètres d'incertitude de modélisation.

Dans un souci de satisfaire aux critères énoncés précédemment (sens physique, transfert des distributions ...), on a retenu la seconde approche qui permet de mieux appréhender le transfert de l'information au cours des différentes constructions de surfaces. 


\section{Surface de rébonse pour le champ cinématique de la Houle}

\subsection{Principe du calcul quasi statique.}

Le calcul d'efforts dus à la houle et aux courants sur une barre immergée de structure "offshore" sous des conditions environnementales sévères est basé sur les équations de Morison [5]. Il s'agit d'une formulation de type énergétique de linteraction fluide-structure. Ainsi le calcul en conditions extrêmes, de type statique, est basé sur un calcul d'efforts issus des champs cinématiques et tient son appellation de quasi statique de cette dualité. La connaissance du champ cinématique au point considéré est donc indispensable. La formulation de ce chargement réparti étant par ailleurs non linéaire, lanalyse du transfert du champ cinématique s'avère délicate. On a ainsi recours à des surfaces de réponse.

\subsection{Surface de réponse pour le champ de vitesse de la houle d'Airy.}

Parmi les modèles relatifs aux champs cinématiques, celui de la houle de Stokes s'avère suffisant pour un calcul en mers profondes. Ce modèle correspond à une solution particulière de léquation hydrodynamique de Navier-Stokes sous l'hypothèse de propagation d'une onde plane et progressive, avec mouvement du fluide irrotationnel à orbites ouvertes. Il permet de caractériser de plus en plus finement la cinématique de la houle par addition de $\mathrm{n}$ champs potentiels correspondant chacun à un ordre d'approximation. Le premier ordre est aussi appelé houle d'Airy et permet ici d'illustrer simplement le modèle formel géométrique mis en place pour la surface de réponse du champ cinématique.

Le potentiel de vitesse s'écrit alors, $\Phi_{\text {airy }}=R_{e}\left(-\frac{g H}{2 \sigma} \frac{\cosh (k d z)}{\cosh (k d)} j \exp j\left(k l x-\psi_{t}\right)\right)$

$\mathrm{H}$ et $\mathrm{k}$, respectivement hauteur et nombre d'onde de la houle, sont les deux variables de base du problème. Compte tenu de la physique de la houle (limitation en cambrure) ces variables ne sont pas indépendantes. La pulsation de la houle $\sigma$, égale à $2 \pi / \mathrm{T}$, est reliée à $\mathrm{k}$ par lintermédiaire de l'équation de dispersion $\left(\mathrm{k} \tanh (\mathrm{kd})=4 \pi^{2} / \mathrm{g} \mathrm{T}^{2}\right)$.

Les paramètres géométriques $\mathrm{x}$ et $\mathrm{z}$ ont été adimensionnés afin de positionner la vague par rapport à la structure. Ainsi, dans le plan de la vague $(0, \overrightarrow{O U}, \overrightarrow{O Z})$, avec $\mathrm{O}$ au niveau moyen, $\overrightarrow{\mathrm{OU}}$, direction de la houle et $\overrightarrow{\mathrm{OZ}}$, axe vertical ascendant,

- $\mathrm{x}=U /$, abscisse adimensionnelle suivant $O U$ et 1 , largeur de la structure $-z=Z / d$, cote adimensionnelle et $d$, profondeur d'eau.

La pseudo-phase $\psi_{\mathrm{t}}\left(\psi_{\mathrm{t}}=\sigma \mathrm{t}+\varphi\right)$ introduit quant à elle la dépendance temporelle. En fait, elle n'est présente que dans les fonctions sinusoïdales et on peut interpréter son influence comme un changement de repère, par translation, suivant la direction de la houle et dont l'origine fluctue avec $\sigma$. Ceci revient à donner un aléa sur la position de la vague par rapport à la structure. Son rôle peut donc être distingué de celui de $\mathrm{x}$ et $\mathrm{z}$. 
Le vecteur vitesse étant dans le plan de la vague, on peut l'écrire sous la forme géométrique,

$$
\overrightarrow{\mathrm{V}}_{\text {airy }}=\#\left(\mathrm{O}, \lambda_{1}\right) \mathrm{O} \Re\left(\overrightarrow{\mathrm{OV}}, \alpha_{1}\right) \cdot \overrightarrow{\mathrm{OU}}
$$

avec $\mathbb{F}\left(\mathrm{O}, \lambda_{1}\right)$ et $\mathfrak{R}\left(\overrightarrow{\mathrm{OV}}, \alpha_{1}\right)$, transformations géométriques, respectivement homothétie de centre $O$, de rapport $\lambda_{1}$ et rotation d'axe $\overrightarrow{O V}=\overrightarrow{O U} \wedge \overrightarrow{O Z}$, d'angle $\alpha_{1}$.

$$
\begin{aligned}
& \tan \left(\alpha_{1}\right)=\tanh (k d z) \tan \left(k l x-\psi_{t}\right) \\
& \text { et } \quad \lambda_{1}=\left\|V_{\text {airy }}\right\|=\frac{H}{2} \sqrt{\frac{g}{d}} \frac{\cosh (k d z)}{\cosh (k d)} \sqrt{\frac{k d}{\tanh (k d)}} \sqrt{1-\frac{\sin ^{2}\left(k l x-\psi_{t}\right)}{\cosh ^{2}(k d z)}}
\end{aligned}
$$

En fait, l'étendue et les caractéristiques statistiques du nombre d'onde $\mathrm{k}$ permettent d'envisager une approximation de Taylor au premier ordre du vecteur $\vec{W}(\mathrm{k})=\Re\left(\overrightarrow{\mathrm{OV}}, \alpha_{1}(\mathrm{k})\right) \overrightarrow{\mathrm{OU}}$ autour de la moyenne $\overline{\mathrm{k}}$ de $\mathrm{k}$, et, après normalisation,

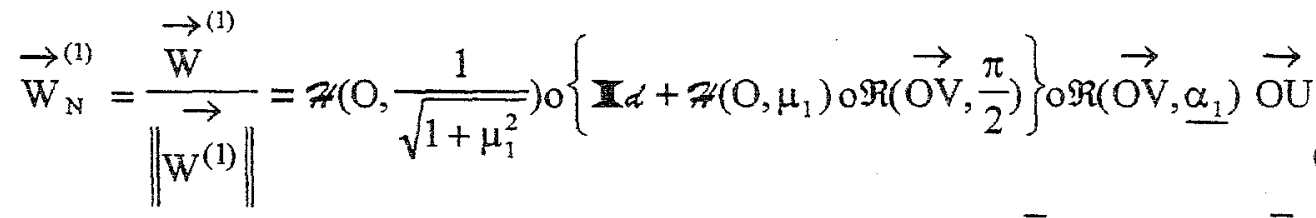

avec $\quad \alpha_{1}^{\prime}$, valeur de la dérivée de $\alpha_{1}$ par rapport à $\mathrm{k}$ en $\mathrm{k}=\overline{\mathrm{k}}$ et $\mu_{1}=(\mathrm{k}-\overline{\mathrm{k}}) \alpha_{1}^{\prime}$. On mesure alors la qualité de cette approximation par le cosinus directeur entre ces deux vecteurs donc ici par leur produit scalaire (3).

$$
\cos (\beta)=<\vec{W}_{N}^{(1)}, \vec{W}>=\frac{\cos \left(\alpha_{1}-\alpha_{1}\right)+\mu_{1} \sin \left(\alpha_{1}-\alpha_{1}\right)}{\sqrt{1+\mu_{1}^{2}}}
$$

Cela revient à mesurer, dans le plan de la vague, la distance entre le point repéré sur le cercle de rayon unitaire par l'angle $\alpha_{1}-\underline{\alpha_{1}}$ et la droite d'équation $\left(U+\mu_{1} Z=\right.$ $0)$. La figure 1 présente ce cercle et les droites obtenues à partir de simulations effectuées pour le champ de Frigg en mer du Nord ; le cosinus directeur est très voisin de 1 pour $\mathrm{k}$ variant sur son support de plus ou moins deux fois l'écart type autour de sa valeur moyenne. L'approximation de Taylor (2) est donc adaptée ici.

Figure 1. Variations de la distance $N-(D)$ avec les fluctuations de $\mathrm{k}$.

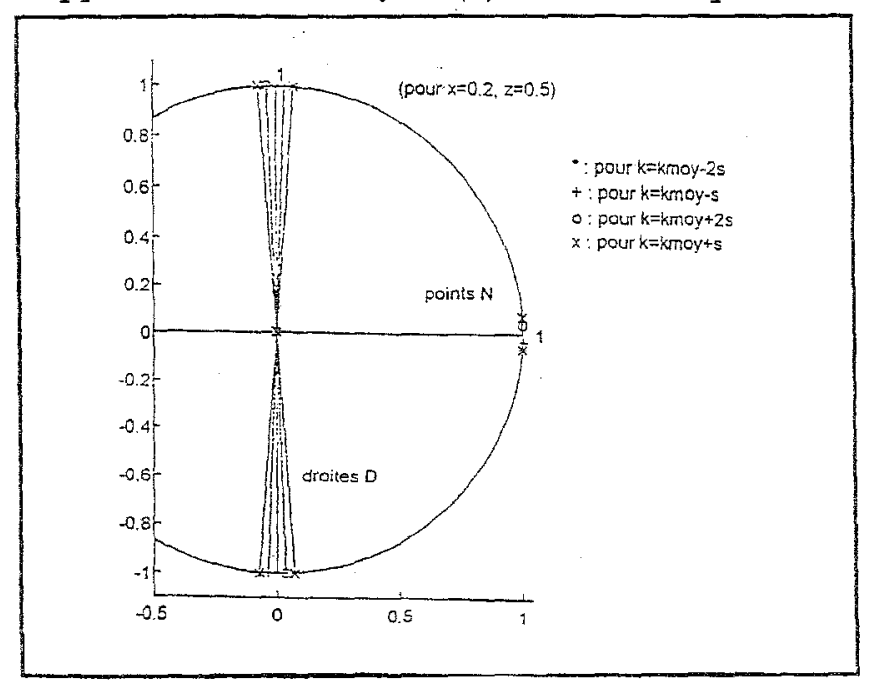


On déduit finalement une approximation du vecteur vitesse en surface de réponse,

$$
\vec{V}_{\text {airy }} \approx \vec{V}_{\text {airy }}^{(1)}=a \vec{A}+b \vec{B}
$$

avec $\quad \vec{A}$ et $\vec{B}$, vecteurs déterministes, unitaires, orthogonaux définis par,

$$
\begin{aligned}
& \vec{A}=\Re\left(\overrightarrow{O V}, \alpha_{1}\right) \overrightarrow{O U}, \vec{B}=\Re\left(\overrightarrow{O V}, \frac{\pi}{2}\right) \vec{A} . \\
& a, b \text {, fonctions aléatoires et, } a=\frac{\lambda_{1}}{\sqrt{1+\mu_{1}^{2}}}, b=\mu_{1} a
\end{aligned}
$$

$\mu_{1}$ étant de moyenne 0 et à support étroit, $b$ est petit par rapport à $a . \vec{A}$ représente donc la direction principale du vecteur vitesse et $\vec{B}$ décrit les petites fluctuations de direction autour de $\overrightarrow{\mathrm{A}}$. Ainsi, on considère $\lambda_{1}$ comme lintensité de la vitesse. Par ailleurs dans l'écriture de $\lambda_{1}$, on peut distinguer le rôle des indices en écrivant : $\lambda_{1}(x, z)=\lambda(0, z) D(x, z)$. En fait, les fluctuations de $D$ sur le support de $k$ sont faibles (coef. de variation inf. à $1 \%$ ) et $D$ peut se réduire à $D$ (valeur de $D$ en $\bar{k}$ ).

$$
\text { Ainsi } \lambda_{1}(x, z)=\lambda(0, z) \underline{D}(x, z) \text { avec } D(x, z)=\sqrt{1-\frac{\sin ^{2}\left(\overline{k l x}-\psi_{t}\right)}{\cosh ^{2}(\overline{k d} z)}}
$$

La dépendance en $\mathrm{x}$ étant présente seulement dans cette fonction, la structure stochastique du champ de vitesse sera gouvernée par des fonctions profils.

Afin d'illustrer ce résultat, une simulation a été conduite à partir d'une base de données issue de l'analyse de mesures de mer du Nord (champ de Frigg) en implémentant une technique d'échantillonnage de type hypercube [6]. Le tracé de la figure 2 est obtenu pour une position de la vague en début de structure et illustre le résultat précédent au travers des tracés des densités de probabilité de $\lambda_{1}$ en fonction de $\mathrm{x}$, pour les cotes $0.2,0.8,1$ (respectivement positions au fond, au creux des plus grandes vagues et au niveau moyen).

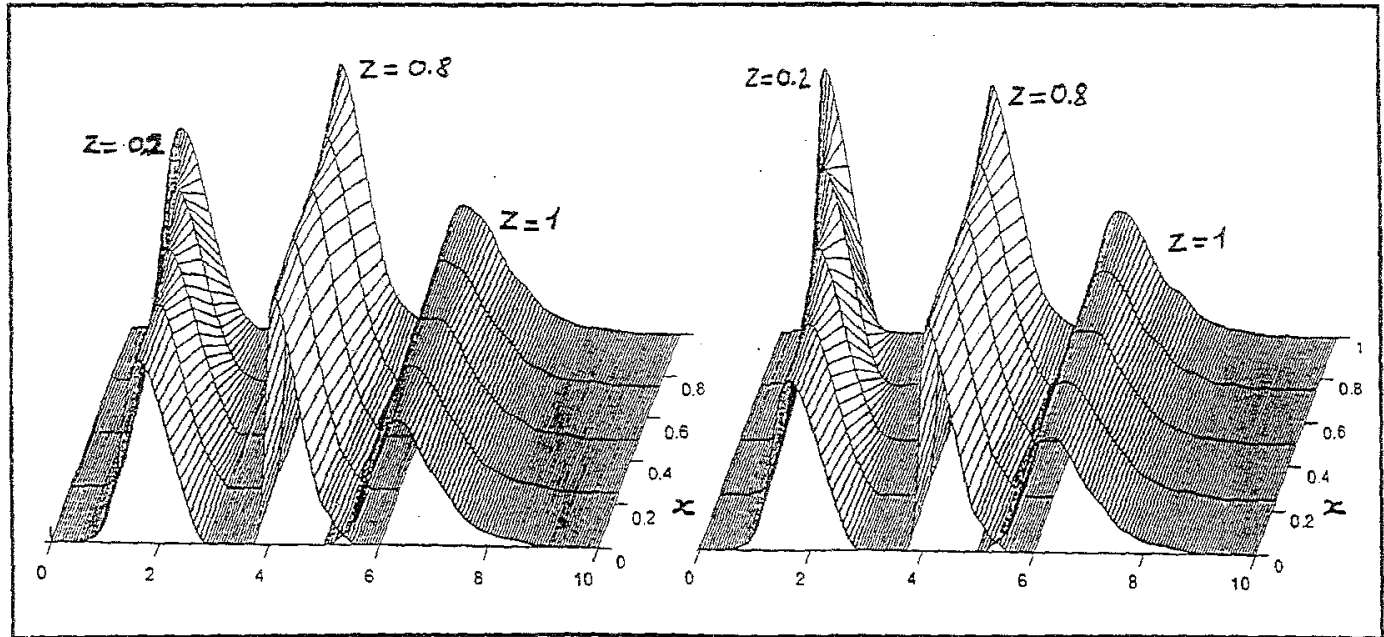

Figure 2. Tracé tridimensionnel des densités de probabilité de $\lambda_{1}[\mathrm{~m} / \mathrm{s}]$.

Le résultat sans approximation (à gauche) est juxtaposé à celui obtenu par l'approximation linéaire (3). 
La seule différence se situe en des positions profondes, où les densités de distribution sont "plus fines". A cette profondeur, les efforts sont plus faibles et cette approximation reste adaptée au calcul d'efforts qui viendra à l'étape suivante.

\subsection{Généralisation à la houle de Stokes au $\mathbf{n}^{\text {ième }}$ ordre.}

Pour la houle de Stokes au $n^{\text {ieme }}$ ordre on a, $\vec{V}_{n}=\vec{V}_{1}+\sum_{i=1}^{n} \vec{V}_{i}$ et on montre de la même manière que $\vec{V}_{i}$ s'écrit sous la forme $\vec{V}_{i}=\mathscr{H}\left(O, \lambda_{i}\right) \circ \Re\left(\overrightarrow{O V}, \alpha_{i}\right) \cdot \overrightarrow{O U}$. On obtient alors une approximation de Taylor, $\vec{V}_{i} \approx \vec{V}_{i}=a_{i} \vec{A}_{i}+b_{i} \vec{B}_{i}$ où $\overrightarrow{A_{i}}=\mathfrak{R}\left(\overrightarrow{O V}, \underline{\alpha_{i}}\right) \cdot \overrightarrow{O U}, \overrightarrow{B_{i}}=\Re\left(\overrightarrow{O V}, \frac{\pi}{2}\right) \cdot \overrightarrow{A_{i}}, a_{i}$ et $b_{i}$ champs stochastiques

Et enfin, par projection sur les vecteurs déterministes du premier ordre, puis par sommation, on déduit la surface de réponse pour la houle de Stokes au $n^{\text {ième }}$ ordre,

$$
\vec{V}_{n}^{\text {ième }} \approx \vec{V}_{n}^{\text {ième }}=a_{(n)} \vec{A}+b_{(n)} \vec{B}
$$

où $a_{(n)}=\sum_{i=1}^{n}\left(a_{i} \cos \underline{\Delta}_{i}-b_{i} \sin \underline{\Delta_{i}}\right)$ et $b_{(n)}=\sum_{i=1}^{n}\left(a_{i} \sin \Delta_{i}+b_{i} \cos \Delta_{i}\right)$

avec $\underline{\Delta_{i}}=\underline{\alpha_{i}}-\underline{\alpha_{1}}$ et on montre de plus, $\underline{\Delta_{i}} \approx(\mathrm{i}-1) \underline{\alpha_{1}}$.

L'accélération se déduit alors par différentiation de (4).

La surface de réponse (4) permet d'observer que la prise en compte d'ordres supplémentaires dans le modèle de Stokes revient à ajouter une contribution faisant un angle $\alpha_{1}$ par rapport à la contribution précédente. $a_{(1)}$ et $b_{(1)}$ correspondent aux champs stochastiques $a$ et $b$ définis au paragraphe précédent. On a vu que la structure stochastique de $a$ était gouvernée par des fonctions profils. On peut donc juger de l'intérêt d'introduire un ordre d'approximation supplémentaire par la comparaison des contributions à lintensité de la vitesse en temps que fonctions profils à projeter sur la direction principale.

Pour le champ de Frigg en mer du Nord, par 100 mètres de profondeur d'eau, cette projection est inutile car il apparait, par la comparaison des profils, que le premier ordre prédomine. Ceux-ci sont donnés en figure 3 pour plusieurs grandeurs statistiques représentatives à savoir la moyenne et les valeurs situées de part et d'autre à plus ou moins une et deux fois l'écart type.

Il est à noter que, avec la même approche, il est possible de montrer que les fluctuations de la direction angulaire $\alpha$ sont gouvernées par le paramètre directionnel $x$.

Ces caractérisations stochastiques de l'intensité et de l'angle permettent d'envisager un calcul numérique plus aisé et mieux maîtrisé des efforts sur une barre immergée [6].

On a montré par ailleurs que cette représentation géométrique peut être étendue à une description, par processus gaussien, d'un état de mer [6]. 


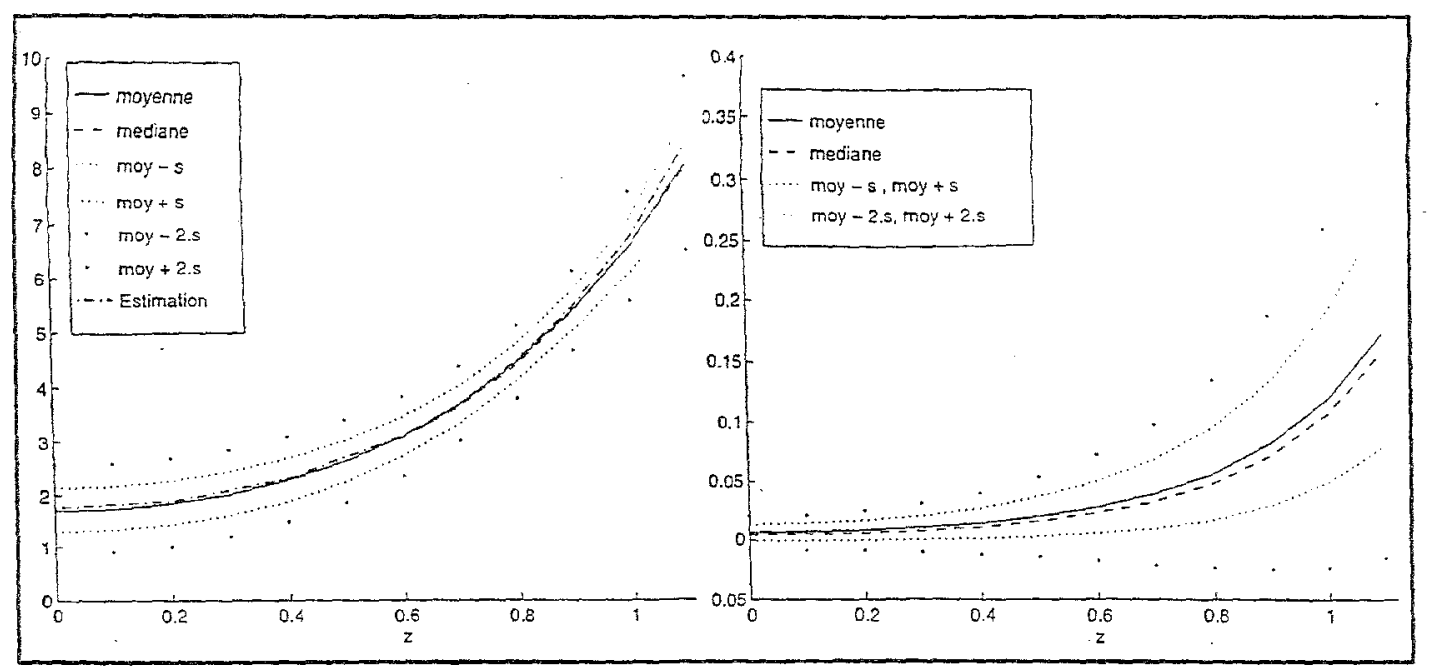

Figure 3. Profils de $\lambda_{1}$ et de $\lambda_{2}$ pour le Champ de Frigg $[\mathrm{m} / \mathrm{s}]$

\section{Conclusion - discussion sur la surface de réponse en vittesse.}

La surface de réponse obtenue permet de caractériser la structure stochastique du champ cinématique. Les fluctuations de lintensité du vecteur vitesse sont caractérisées par des fonctions profil qui facilitent le choix du niveau de complexité à introduire dans le modèle de Stokes, en fonction du site.

Cette caractérisation va permettre de discuter les hypothèses d'interaction houlecourants sur certains sites (golfe de Guinée par exemple), là où les techniques de linéarisation ou de perturbations ne sont plus suffisantes. La difficulté réside alors dans la validation de modèles analytiques d'interaction en conditions sévères.

Remerciements. Ce travail sintègre dans le programme "Requalification des structures pétrolières offshore existantes" du CLAROM dans lequel participent le Bureau Veritas, Ifremer, Géodia, Bouygues Offshore, EDF, Principia ainsi que Elf et Total pour les études de cas.

\section{Références bibliographiques.}

[1] O. Ditlevsen \& H.O. Madsen : Structural Reliability Methods, WileyInterscience-Europe, (en impression - Jan. 1996).

[2] R. Mead \& D.J. Pike : A review of response surface methodology from $a$ biometric viewpoint, Biometrics 31, pp. 803-851, 1975.

[3] J. Labeyrie : Response Surface Methodology in Structural Reliability, Book "Probabilistic Mechanics", Klouwer Ed, 1996.

[4] V. Bouyssy \& R. Rackwitz : Approximation of non-normal responses for drag dominated offshore structures, 6th IFIP WG7.5, "Reliability and Optimisation of structural system", $n^{07}, 1995$.

[5] J.R. Morison, M.P. O'Brien, J.W. Johson \& S.A. Schaff : The forces exerted by surfaces waves on piles, Petrol. trans. vol. 189, pp. 149-154, 1950.

[6] J. Labeyrie \& F. Schoefs : A Formal Geometrical Modelling of Wave Actions for Structural Reliability, 14th int. conf. on Offshore Mechanics and Arctic Engineering, (O.M.A.E'95), Copenhagen, pp. 85-90, 1995. 